\title{
Clinical assessment of DSM-IV anxiety disorders in fragile $X$ syndrome: prevalence and characterization
}

\author{
Lisa Cordeiro • Elizabeth Ballinger • Randi Hagerman • \\ David Hessl
}

Received: 18 May 2010 /Accepted: 10 November 2010 /Published online: 3 December 2010

(C) The Author(s) 2010. This article is published with open access at Springerlink.com

\begin{abstract}
Fragile X syndrome (FXS) is the most common form of inherited intellectual disability (ID). Anxiety and social withdrawal are considered core features of the FXS phenotype, yet there is limited diagnostic evidence of the prevalence of formal anxiety disorders in FXS. This study assessed the prevalence of anxiety disorders in a sample of 58 males and 39 females with FXS (ages 5.0-33.3 years). Participants' parents completed the Anxiety Disorders Interview Schedule (ADIS-IV), a clinical interview based on DSM-IV criteria, and the Anxiety Depression and Mood Scale (ADAMS), a psychiatric disorders screening instrument normed in ID. We conducted cognitive (IQ) and autism (AUT) assessments and surveyed medication use. Despite a high rate of psychopharmacological treatment, $86.2 \%$ of males and $76.9 \%$ of females met criteria for an anxiety disorder, with social phobia and specific phobia the most commonly diagnosed. Proband status, gender, and IQ were not significantly related to any anxiety disorders, however
\end{abstract}

L. Cordeiro $\cdot$ E. Ballinger $\cdot$ R. Hagerman $\cdot$ D. Hessl $(\bowtie)$

M.I.N.D. Institute, University of California,

Davis Medical Center,

2825 50th Street,

Sacramento, CA 95817, USA

e-mail: david.hessl@ucdmc.ucdavis.edu

L. Cordeiro

Department of Human Development,

University of California Davis,

Sacramento, CA, USA

R. Hagerman

Department of Pediatrics, University of California Davis,

Sacramento, CA, USA

L. Cordeiro $\cdot$ E. Ballinger $\cdot$ D. Hessl

Department of Psychiatry and Behavioral Sciences,

University of California Davis,

Sacramento, CA, USA significantly higher rates of a few anxiety disorders were found in older age and AUT groups. Significant correlations between ADIS diagnoses and ADAMS scores provided crossvalidation of instruments, indicating that the ADIS is suitable for use in FXS. A greater percentage of our sample met criteria for most anxiety disorders than has been reported in other ID groups or the general population. The rate of anxiety compared to general ID suggests that the FMRI full mutation confers an especially high risk for these disorders, regardless of factors commonly associated with FXS clinical involvement. A thorough clinical assessment and treatment of anxiety should be included in the FXS standard of care.

Keywords Fragile X syndrome - Anxiety · Social phobia . Specific phobia $\cdot$ Intellectual disability

$\begin{array}{ll}\text { Abbreviations } \\ \text { FXS } & \text { Fragile X syndrome } \\ \text { FMR1 } & \text { Fragile X mental retardation 1 gene } \\ \text { FMRP } & \text { Fragile X mental retardation protein } \\ \text { ID } & \text { Intellectual disability } \\ \text { DD } & \text { Developmental delay } \\ \text { ASD } & \text { Autism spectrum disorder } \\ \text { AUT } & \text { Autism } \\ \text { SM } & \text { Selective mutism } \\ \text { WS } & \text { Williams syndrome } \\ \text { ADIS } & \text { Anxiety Disorders Interview Schedule } \\ \text { ADAMS } & \text { Anxiety Depression and Mood Scale } \\ \text { DS } & \text { Down syndrome }\end{array}$

\section{Introduction}

Fragile X syndrome (FXS) is the leading cause of genetically inherited intellectual disability (ID). FXS results from an 
expansion of CGG repeats at Xq27.3 in the fragile X mental retardation gene (FMRl) on the long arm of the $\mathrm{X}$ chromosome. The frequency of the full mutation allele is approximately one in 2,500 (Hagerman 2008) although the affected prevalence has been estimated to be one in 3,600 (Crawford et al. 2002; Fernandez-Carvajal et al. 2009) because some individuals with the full mutation, such as females, do not have significant ID. The expansion leads to a reduction or elimination of FMR1 protein (FMRP) production known to be essential for normal brain function and growth (Bassell and Warren 2008). Due to the presence of a second $\mathrm{X}$ chromosome, females can be less cognitively impaired than males but are vulnerable to behavioral and emotional problems (Lachiewicz 1992, 1995; Lachiewicz and Dawson 1994), as well as math deficits (Murphy 2009; Lachiewicz et al. 2006). Variability in functioning of the FMR1 gene (Xactivation ratio in females, FMRP expression, methylation, etc.) leads to heterogeneity in clinical presentation, among both males and females with FXS (Loesch et al. 2004).

The behavioral phenotype of FXS is characterized by mild to severe ID, anxiety, social deficits, hyperarousal, abnormalities in communication, abnormal sensory reactivity, gaze aversion, inattention, impulsivity, aggression, and hyperactivity (Sudhalter et al. 1990; Cohen et al. 1991; Reiss and Freund 1992; Hessl et al. 2001). Anxiety has been recognized as a prominent feature of FXS, yet the severity and pervasiveness of clinical anxiety disorders in this population have not been studied. Despite the lack of diagnostic information, anxiety symptoms are frequently treated with medications (Berry-Kravis and Potanos 2004).

In the general population, anxiety disorders are among the most common psychiatric disorders, occurring in $2.4 \%$ to $10.7 \%$ of children (Fergusson et al. 1993; McGee et al. 1990; Costello et al. 2003). As part of an epidemiological study, the National Institutes of Mental Health (NIMH) developed a DSM-III-R criteria-based interview (the Diagnostic Interview Schedule for Children, DISC) to assess the presence of psychiatric disorders and found that $9.8 \%$ of children met criteria for an anxiety disorder $(N=1,289,9-17$ years) (Shaffer et al. 1996). The most common anxiety disorder was social phobia, followed by overanxious disorder (analogous to generalized anxiety disorder in the DSM-IV) and separation anxiety $(4.5 \%, 3.1 \%$, and $2.3 \%$, respectively). In a national survey of households $(N=9,282)$, the National Comorbidity Survey (NCS-R) found that the median age of onset for anxiety disorders was 11 years, with half of all cases occurring between 6 and 21 years old (Kessler et al. 2005). Many studies have reported that girls are at higher risk for an anxiety disorder, at any age, compared with males (Fergusson et al. 1993; McGee et al. 1990; Costello et al. 2003).

The clinical assessment of anxiety in individuals with ID, including those with FXS, can be particularly difficult. Though psychiatric problems in ID are common, they are not often accurately identified (Reiss and Valenti-Hein 1994). Clinical diagnosis of psychiatric problems often relies upon verbal expression and a degree of personal insight, which can be lacking in ID. Many treatments, interventions, and allocation of services are tied to the clinical diagnosis of these disorders. Therefore, individuals with ID who are not properly diagnosed may not receive the supports and treatment they need. The most recent version of the DSM suggests that adaptations to diagnostic criteria made for children are appropriate for individuals with ID.

Despite the complexity of psychiatric diagnosis among those with ID, the risk for psychopathology in this population is high. It is estimated that individuals with ID are two to four times more likely than the general population to experience psychiatric problems (Eaton and Menolascino 1982). Dekker and Koot (2003) found that $38.6 \%$ of individuals with idiopathic ID met criteria for a psychiatric disorder.

Similar to the general population, anxiety disorders are prevalent among individuals with ID. Using the DISC to measure DSM-IV disorders ( $N=474$, ages $7-20$ years), Dekker and Koot (2003) reported anxiety disorders in $21.9 \%$ of children with ID. Specific phobia was the most common $(17.5 \%)$, followed by obsessive-compulsive disorder (OCD; 2.7\%) and social phobia (2.5\%). Neither generalized anxiety disorder (GAD) or posttraumatic stress disorder (PTSD) was found in the sample. In contrast to general population data, there was no relationship between age and anxiety disorders, except for OCD. Myers and Pueschel (1991) found that $22.1 \%$ of individuals with Down syndrome (DS) met DSM-III criteria for a psychiatric disorder. However, anxiety disorders were not common in those with DS: only $1 \%$ of participants were diagnosed with phobias and $0.8 \%$ with OCD.

Other genetically caused neurodevelopmental disorders, such as Williams syndrome and $22 \mathrm{q}$ deletion syndromes, exhibit higher rates of anxiety compared to the general population. Previous studies of 22q deletion syndromes have reported rates of anxiety disorders ranging from $32 \%$ to $60.7 \%$ (Dekker and Koot 2003; Myers and Pueschel 1991; Gothelf et al. 2007; Prasad et al. 2008). The most common diagnoses were OCD, specific phobia, and social phobia (32.6\%, $32.6 \%$, and $18.6 \%$, respectively). Multiple studies have described the behavioral and emotional characteristics of individuals with WS (Einfeld et al. 2001; Dykens 2003; Gosch and Pankau 1997). Both WS and FXS share the features of cognitive deficits and anxiety. While social withdrawal is associated with FXS, the WS phenotype is described as extremely social and outgoing. In studies using a DSM-IV-based interview (Leyfer et al. 2006a, b), 62.1\% of individuals with WS were diagnosed with at least one anxiety disorder, with specific phobia being the most common (56.1\%) and social phobia occurring in only $2.3 \%$ of the sample. Taken together, these studies show that rates 
and types of anxiety disorders vary among genetic disorders causing ID. However, anxiety is more prevalent in these genetic conditions compared to the general population.

Anxiety symptoms have long been recognized as a prominent component of the FXS phenotype. In a recent national survey of 1,492 children with FXS, parents reported that $70 \%$ of males and $56 \%$ of females with FXS had been treated for anxiety symptoms or received a formal anxiety diagnosis (Bailey et al. 2008). A review of the literature in FXS shows reliance upon questionnaires and behavior checklists, many of which were not developed for use in individuals with ID. Anxiety and social withdrawal symptoms are commonly reported in parent-report questionnaire studies of children with FXS.

Lachiewicz (1992) used a parent-report questionnaire, the Child Behavior Checklist (CBCL), in a sample of 38 females (ages 4-11 years) with FXS. Almost half (47\%) of the girls scored above the 98th percentile on the social withdrawal subscale. More girls with average IQ (64\%) than those with borderline IQ (28\%) or IQs below 70 (46\%) scored above the 98th percentile. This pattern could have been due to an artifact of the measure since the CBCL version used had not been developed or normed in ID. Similar findings were reported using the Conners Rating Scale to compare a group of females with FXS and a group of females with developmental delay (DD) without FXS. There was a significant difference between the percentage of girls with t-scores above the 98th percentile in the DD (2\%) and FXS (23\%) groups (Lachiewicz and Dawson 1994). Similar to the CBCL findings, more girls with FXS who had normal or borderline IQ had t-scores above the 98th percentile compared to those with IQ scores below 70 .

A few studies have used diagnostic criteria to measure psychiatric problems in FXS. For example, Freund et al. (1993) assessed a group of FXS females $(\mathrm{N}=17)$ and nonFXS age-matched controls $(\mathrm{N}=17)$ (ages 4-27 years old) for the presence of DSM-III-R psychiatric disorders. Using the DICA-P interview, there was a greater frequency of avoidant disorder (11 of 17) and mood disorders ( 8 of 17) in the FXS group compared to controls. Two of the 17 females with FXS met criteria for overanxious disorder, compared to none of the controls. Further, parents and teachers rated the FXS group as significantly more withdrawn and depressed compared to control participants. It is noteworthy that this study used a behavior checklist normed in both learning disability and DD populations, as well as a semi-structured interview and found no association between IQ and anxiety, as reported in the Lachiewicz studies.

The goal of the present study was twofold. First, we sought to measure the prevalence and characterize the types of anxiety problems in individuals with FXS using a diagnostic clinical interview and questionnaire normed in ID, while addressing adjustments in the diagnostic formu- lation related to children and ID. Second, we examined factors that may be associated with the presence of anxiety disorders in individuals with FXS. Third, we compared rates of anxiety disorders in FXS to those reported in the general population, in ID, and in another specific genetic syndrome manifesting anxiety, Williams syndrome.

\section{Method}

\section{Participants}

Participants were 58 males (ages 5.0-26.7 years, $M=13.07$, $\mathrm{SD}=5.60$ ) and 39 females (ages 5.5-33.3 years, $M=12.35$, $\mathrm{SD}=6.17$ ) with a confirmed diagnosis of FXS (see Table 1). The majority of participants were the first to be identified with FXS in their family: $64.9 \%$ were probands ( 52 males, 11 females). The remainder of the sample was identified by cascade DNA testing. Race and ethnicity data were collected in accordance with NIMH funded project requirements. The majority of the sample was Caucasian (78.4\%) and not Hispanic or Latino (64.9\%).

\section{Measures}

The Anxiety Disorders Interview Schedule for DSM-IV: Parent Report Version (ADIS-IV) (Silverman and Albano 2004) was administered to measure the presence and severity of current anxiety disorders according to DSM-IV criteria.

Table 1 Description of participants

\begin{tabular}{llll}
\hline & Males & Females & Total \\
\hline Total N & 58 & 39 & 97 \\
Age M & 13.07 & 12.35 & 12.78 \\
SD & 5.60 & 6.17 & 5.81 \\
Range & $5.00-26.71$ & $5.50-33.30$ & $5.00-33.30$ \\
IQ M & 60.45 & 77.20 & 67.18 \\
SD & 15.36 & 20.65 & 19.41 \\
Range & $30-117$ & $40-116$ & $30-117$ \\
ID ${ }^{\text {a status } N(\%)}$ & & & \\
ID (FSIQ<70) & $45(77.6)$ & $13(33.3)$ & $58(59.8)$ \\
Non-ID & $12(20.7)$ & $26(66.7)$ & $38(39.2)$ \\
ASD ${ }^{b}$ diagnosis $N(\%)$ & & & \\
No ASD & $21(36.2)$ & $28(71.8)$ & $49(50.5)$ \\
ASD & $10(17.2)$ & $10(25.6)$ & $20(20.0)$ \\
Autism & $27(46.6)$ & $1(2.6)$ & $28(28.9)$ \\
Proband status $N(\%)$ & & & \\
Proband & $52(89.7)$ & $11(28.2)$ & $63(64.9)$ \\
Non-proband & $6(10.3)$ & $28(71.8)$ & $34(35.1)$ \\
\hline
\end{tabular}

${ }^{\mathrm{a}} I D$ Intellectual Disability, ${ }^{\mathrm{a}} A S D$ Autism Spectrum Disorder 
This measure is a semi-structured interview designed to diagnose a variety of anxiety disorders and allows the rater (the parent) to make dimensional ratings of disorder features. These ratings indicate either the degree of distress or interference the item presents in a person's functioning $(0=$ none to $8=$ very severe $)$. The test-retest reliability is good $(\mathrm{k}=0.73)$ (Silverman et al. 2001). Inter-rater reliability between the parent- and child- report versions of the ADIS for both principal diagnosis and individual anxiety disorders is excellent $(\mathrm{k}=.80-1.0)$ (Lyneham et al. 2007). Administration of the ADIS takes an average of two hours and was completed with the participant's mother $(89.2 \%)$, father (5.4\%) or mother and father combined (5.4\%). The ADIS has been used extensively in published studies of anxiety across many settings and populations (Ross et al. 2007; Panichelli-Mindel et al. 2005; Leyfer et al. 2009; Bodden et al. 2008; Waters et al. 2008), in validation studies (Thaler et al. 2010; Simon and Bogels 2009; Bodden et al. 2009; Leyfer et al. 2006b), as well as a federally-funded pediatric anxiety treatment trial (Compton et al. 2010).

The Anxiety Depression and Mood Scale (ADAMS) (Esbensen et al. 2003) is a 28-item questionnaire used to screen for psychiatric disorders in persons with ID. Behaviors are rated on a 4-point Likert scale ranging from 0 ("not a problem") to 3 ("severe problem"). The ADAMS yields 5 subscale scores: General Anxiety, Social Avoidance, Depression, Manic/Hyperactive and Obsessive/Compulsive Behavior. It was psychometrically evaluated and normed using 265 individuals and validated with 129 psychiatric patients with ID (Esbensen et al. 2003). For the current study, the ADIS and ADAMS were completed by the same respondent (mother or father) within a maximum interval of 14 days.

Intelligence testing was conducted as developmentally appropriate by a trained clinician. Due to the wide age range of participants, measures included tests from the Wechsler Scales of Intelligence: WASI (48.4\%), WPPSI-III (8.6\%), WAIS-III (9.7\%), WISC-III or WISC-IV (16.1\%) and a limited number of non-verbal intelligence tests $(4.3 \%)$. Those with an IQ score below 70 were classified as having an ID.

Assessment for the presence of an autism spectrum disorder (ASD) included the Autism Diagnostic Observation Scale (ADOS-G), the Autism Diagnostic InterviewRevised (ADI-R), DSM-IV criteria and clinical team consensus as previously described (Harris et al. 2008). All diagnostic assessments used to determine ASD status were administered by a trained clinician.

\section{Procedures}

Consent or assent was obtained from all participants (and parents, if applicable) in the study and all procedures were approved by the institutional review board. Participants were seen for 1 to 3 days as part of a larger study of the physiological correlates of anxiety in FXS. A list of current medications was reported by the parents. The ADIS was administered by an experienced, licensed clinical psychologist (D.H.) or graduate level student (L.C.) who had passed reliability training on the instrument. Training for the ADIS began with reviewing the interview, observing both videotaped and live administrations of the interview and assigning diagnoses and severity ratings for each interview. Once trainees had obtained three or more matches on diagnosis and severity, a practice ADIS was administered and videotaped. The videotape was reviewed by a licensed clinical psychologist. Once the trainee and the clinical psychologist matched on diagnoses and severity, the interviewer was permitted to administer the ADIS for the study. Disagreements of diagnosis were resolved by case discussion, with final diagnosis made by the licensed clinical psychologist. One-third of all cases were reviewed as a team.

During the ADIS interview, specific examples were requested of the parent to check for comprehension and ensure proper symptom endorsement. For example, if fear of dogs was endorsed in the specific phobia section, parents were asked to describe the reaction, the last time it occurred, the consistency of the fear, and the degree and type of interference in daily functioning. Parents were also asked whether their child 'reported' fear or if they had 'observed' a reaction or behavior demonstrating fearfulness or distress. The collection of this information allowed for any necessary diagnostic adjustments, as described below.

The information collected during the ADIS interview along with the age, ID classification and autism status of each individual were used to make diagnostic determinations. The DSM-IV-TR notes that in children and adults with ID who function at similar levels, adaptations for children are applicable. As such, in children with or without ID and adults with ID, DSM-IV adaptations for children were followed. For example, specific phobia in children may be expressed as crying, tantrums, freezing or clinging. Further, children may not recognize that the fear is excessive or unreasonable. Standard DSM-IV criteria were used in adults without ID.

Statistical analysis

\section{Possible factors related to anxiety}

Exploratory analyses of the relationships between proband status, autism status, IQ score, ID status, gender and age with both the presence and number of anxiety disorders were conducted in preparation for planned multiple logistic (MLR) and multiple linear (MRA) regressions, respectively. 
Due to a lack of significant relationships in the exploratory analyses, multiple univariate comparisons and a high rate of anxiety disorders in this sample, MLR and MRA were ultimately not conducted.

\section{Cross-validation of interview and questionnaire data}

Spearman's rho correlations were used to assess the association between each anxiety disorder and each ADAMS subscale. Pearson's moment correlations were used to assess the relationship between total number of anxiety disorders diagnosed and ADAMS scores. These relationships were examined to validate the use of the ADIS in FXS.

\section{Comparison of anxiety disorder rates}

Proportion tests (z-tests) (Newcombe 1998a, b) were carried out using the SPSS (v17) Custom Tables module (SPSS Statistics 2007a, b) to determine if the prevalence of anxiety disorders in the current study were significantly different from prevalence in previous studies among the following groups: general population, idiopathic ID, and Williams syndrome. Alpha values were adjusted for multiple comparisons using a Bonferroni correction.

\section{Results}

\section{Anxiety}

Among the entire sample of participants with FXS, 82.5\% met criteria for at least one anxiety disorder and $58.3 \%$ met criteria for multiple anxiety disorders (see Table 2). The majority of participants met criteria for one to three of the nine anxiety disorders assessed. The most common anxiety disorders were specific phobia ( $64.9 \%$ of males and $51.4 \%$ of females), social phobia (34.5\% of males and $39.5 \%$ of females) and SM ( $28.1 \%$ of males and $25.3 \%$ of females). Many more participants had clinical symptoms of anxiety, although they did not meet full diagnostic criteria for the disorder. This demonstrated a range of clinical involvement related to anxiety, among both males and females with FXS (see Figs. 1 and 2 , respectively).

For social phobia only, an additional diagnostic category was examined based on the authors' determination that the screening question ("In social situations, does your child worry that they might do something that will be embarrassing?") was beyond the cognitive or expressive language capacity of most of those with ID. This modification to the criteria and resulting category is hereafter termed "social phobia (adjusted)". Elimination of the screening question criteria (but maintenance of all other criteria) increased the rate of social phobia to $60.3 \%$ of males and $55.3 \%$ of females. This adjustment also resulted in an increased rate of social phobia among participants with ID (from $32.8 \%$ to $69.0 \%$ ). This single modification allowed for a diagnosis of social phobia in individuals who demonstrated clinically significant impairment as a result of social phobia symptoms, but who are not able to verbalize or explain "a worry that they might do something embarrassing."

\section{Use of medications}

Medications were used by $53.6 \%$ of participants. The most common class of medication was selective serotonin reuptake inhibitors (SSRIs), used by $27.8 \%$ of participants $(24.1 \%$ of males and $33.3 \%$ of females). Stimulants and atypical

Table 2 Percentage of individuals with FXS meeting criteria for DSM-IV anxiety disorders

\begin{tabular}{|c|c|c|c|c|c|c|c|c|}
\hline \multirow[b]{2}{*}{ Anxiety type } & \multirow[b]{2}{*}{ Total } & \multicolumn{2}{|l|}{ Gender } & \multicolumn{3}{|c|}{ Autism status } & \multicolumn{2}{|l|}{ ID status } \\
\hline & & Female & Male & No autism & $\mathrm{ASD}^{\mathrm{a}}$ & Autism & Non- ID ${ }^{\mathrm{b}}$ & $\mathrm{ID}^{\mathrm{b}}$ \\
\hline Multiple disorders & $58.3 \%$ & $55.3 \%$ & $60.3 \%$ & $56.3 \%$ & $55.0 \%$ & $64.3 \%$ & $51.4 \%$ & $63.8 \%$ \\
\hline Any disorder & $82.5 \%$ & $76.9 \%$ & $86.2 \%$ & $81.6 \%$ & $85.0 \%$ & $82.1 \%$ & $76.3 \%$ & $87.9 \%$ \\
\hline Separation anxiety & $11.5 \%$ & $18.4 \%$ & $6.9 \%$ & $14.6 \%$ & $10.0 \%$ & $7.1 \%$ & $13.5 \%$ & $10.3 \%$ \\
\hline Social phobia & $36.5 \%$ & $39.5 \%$ & $34.5 \%$ & $39.6 \%$ & $30.0 \%$ & $35.7 \%$ & $43.2 \%$ & $32.8 \%$ \\
\hline Social phobia (adj.) & $58.3 \%$ & $55.3 \%$ & $60.3 \%$ & $58.3 \%$ & $45.0 \%$ & $67.9 \%$ & $43.2 \%$ & $69.0 \%$ \\
\hline Specific phobia & $59.6 \%$ & $51.4 \%$ & $64.9 \%$ & $50.0 \%$ & $65.0 \%$ & $71.4 \%$ & $42.9 \%$ & $70.7 \%$ \\
\hline Panic disorder & $5.4 \%$ & $2.7 \%$ & $7.1 \%$ & $6.4 \%$ & $.0 \%$ & $7.1 \%$ & $2.8 \%$ & $6.9 \%$ \\
\hline Agoraphobia & $12.9 \%$ & $10.8 \%$ & $14.3 \%$ & $11.1 \%$ & $5.0 \%$ & $21.4 \%$ & $5.6 \%$ & $17.9 \%$ \\
\hline GAD & $23.7 \%$ & $18.4 \%$ & $27.3 \%$ & $30.4 \%$ & $15.8 \%$ & $17.9 \%$ & $27.8 \%$ & $21.4 \%$ \\
\hline OCD & $23.7 \%$ & $18.4 \%$ & $27.3 \%$ & $26.1 \%$ & $20.0 \%$ & $22.2 \%$ & $19.4 \%$ & $26.8 \%$ \\
\hline PTSD & $4.3 \%$ & $5.4 \%$ & $3.5 \%$ & $6.5 \%$ & $.0 \%$ & $3.6 \%$ & $8.6 \%$ & $1.7 \%$ \\
\hline Selective mutism & $25.3 \%$ & $21.1 \%$ & $28.1 \%$ & $17.0 \%$ & $25.0 \%$ & $39.3 \%$ & $21.1 \%$ & $27.6 \%$ \\
\hline
\end{tabular}

${ }^{\mathrm{a}} A S D$ Autism Spectrum Disorder, ${ }^{\mathrm{b}} I D$ Intellectual Disability 


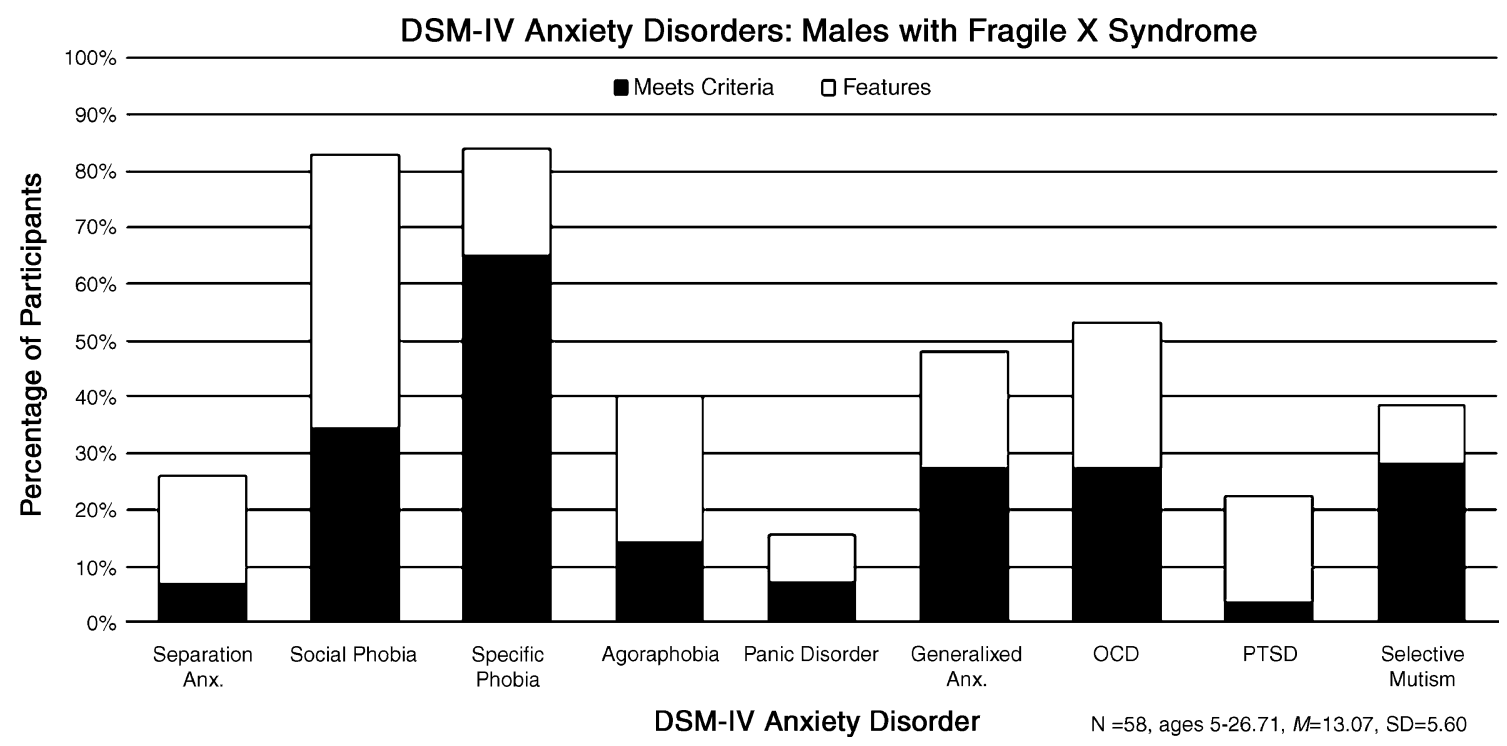

Fig. 1 DSM-IV anxiety disorders: males with fragile X syndrome. Features (open portion of bars) shows the percentage of participants who demonstrated clinically significant symptoms but did not meet formal diagnostic criteria

anti-psychotics were also common $(24.7 \%$ and $20.6 \%$, respectively). A greater percentage of probands (27.0\%) than non-probands $(8.8 \%)$ were using anti-psychotics. A similar pattern was found for the use of anti-psychotics among those with $(27.6 \%)$ and without ID (10.7\%). Among the subset of participants taking medications, more females $(72.2 \%)$ were taking an SSRI than males $(41.2 \%)$. A more detailed description of medication usage is in Table 3.

Possible factors related to anxiety

The relationships between proband status and gender with the presence of any or total number of anxiety disorders were not significant $(p=.07$ and $p=.18$, respectively). Social phobia and PTSD were significantly more common in individuals over age 18 than among children $(p<.05)$. A greater percentage of participants with ID met criteria for all anxiety disorders measured, except for Separation Anxiety, Social Phobia (unadjusted), GAD and PTSD. Comparison of FXS+ID and FXS-ID groups revealed a higher rate of specific phobia and social phobia (adjusted) among those with a below average IQ $(p<.05)$. Note that there was not a significant difference in the rate of social phobia among FXS+ID and FXS-ID when the aforementioned adjustment to criteria was not made. The percentage of participants who met criteria for an anxiety disorder

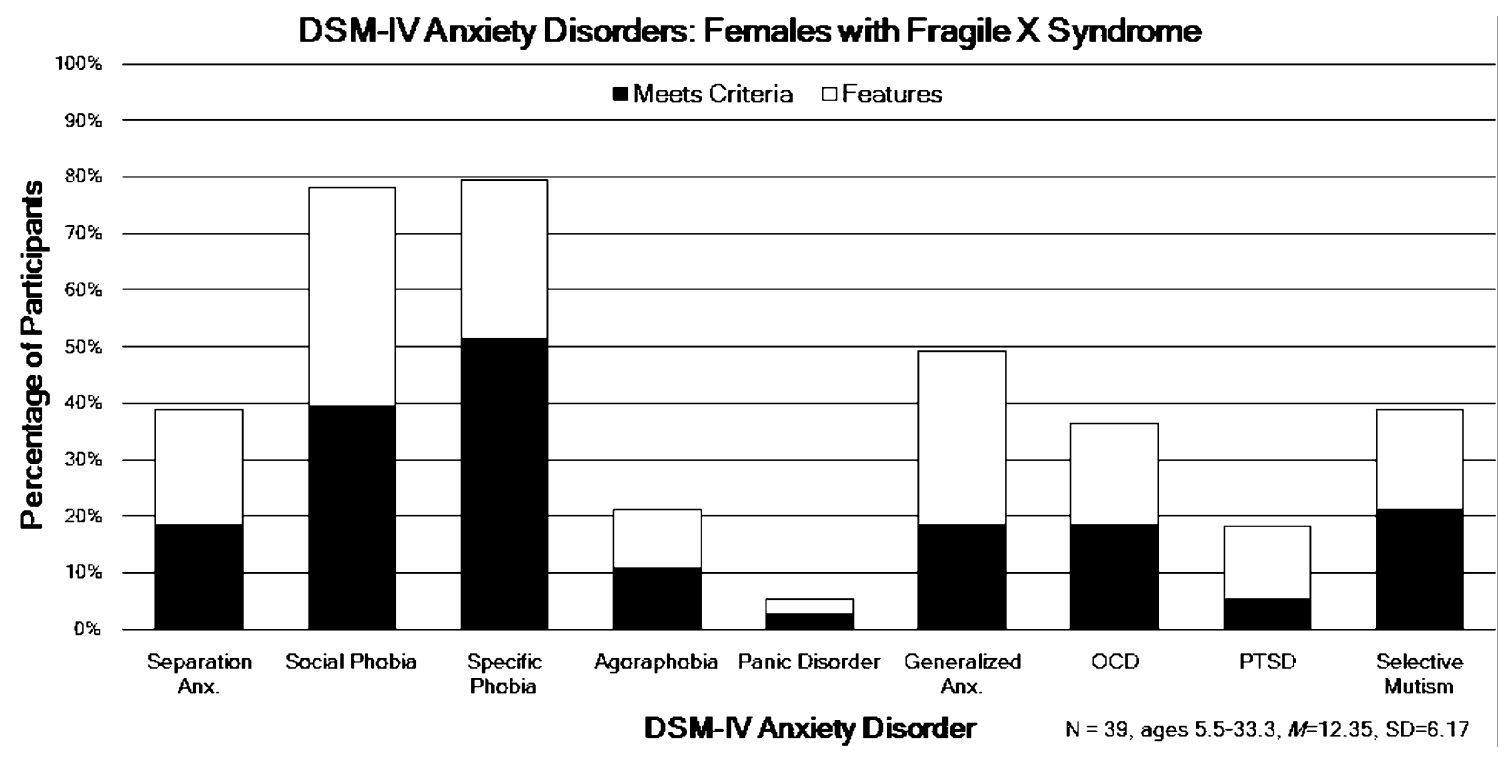

Fig. 2 DSM-IV anxiety disorders: females with fragile X syndrome. Features (open portion of bars) shows the percentage of participants who demonstrated clinically significant symptoms but did not meet formal diagnostic criteria 
Table 3 Medication use among males \& females with FXS

\begin{tabular}{lrrr}
\hline & Total & Gender & \\
\cline { 3 - 4 } & & Female & Male \\
Medication type & & $33.3 \%$ & $24.1 \%$ \\
\hline SSRI & $27.8 \%$ & $2.6 \%$ & $8.6 \%$ \\
Anti-depressant & $6.2 \%$ & $15.4 \%$ & $24.1 \%$ \\
Anti-psychotic & $20.6 \%$ & $17.9 \%$ & $29.3 \%$ \\
Stimulant & $24.7 \%$ & $.0 \%$ & $12.1 \%$ \\
AED/Anti-convulsant & $7.2 \%$ & $2.6 \%$ & $5.2 \%$ \\
SNRI & $4.1 \%$ & $15.4 \%$ & $20.7 \%$ \\
Miscellaneous & $18.6 \%$ & $.0 \%$ & $1.7 \%$ \\
Sedative & $1.0 \%$ & $.0 \%$ & $1.7 \%$ \\
Anti-histamine & $1.0 \%$ & $.0 \%$ & $1.7 \%$ \\
Anti-anxiety & $1.0 \%$ & $53.8 \%$ & $41.4 \%$ \\
No medications & $46.4 \%$ & .9 & 1.4 \\
Total medications & 1.2 & & \\
\hline
\end{tabular}

across autism groups did not follow a consistent pattern. Some anxiety disorders, such as Specific Phobia and Social Phobia (adjusted), occurred more frequently among participants with Autism. Significantly more participants with an ASD were diagnosed with SM $(\mathrm{p}<.05)$, despite accounting for communication deficits among those with an ASD.

Cross-validation of interview and questionnaire data

The ADAMS Social Avoidance subscale was significantly correlated with two social anxiety disorders: Social Phobia $(\mathrm{rho}=.263, \mathrm{p}=.02)$ and Selective Mutism $(\mathrm{rho}=.421, \mathrm{p}=.001)$. The ADAMS OCD subscale was significantly correlated with an ADIS OCD diagnosis $(r h o=.252, p=.04)$. The ADAMS General Anxiety subscale was significantly correlated with many ADIS diagnoses $(\mathrm{rho}=.251$ to $.338, \mathrm{p}<.05)$ and total number of ADIS anxiety diagnoses $(\mathrm{r}=.508, \mathrm{p}<.001)$. No significant relationships were found between the ADAMS Depression subscale and the presence of any ADIS diagnosis.

\section{Comparison of anxiety in FXS with other groups}

Prevalence rates of anxiety disorders were compared to the rates in a group with idiopathic ID, the general population, and a group of individuals with Williams syndrome (WS) (see Table 4). The comparison of FXS+ID to idiopathic ID included data from the largest $(\mathrm{N}=474)$ epidemiological study of psychiatric disorders among children (ages 6-18 years) with idiopathic ID using DSM-IV criteria (Dekker and Koot 2003). The comparison of FXS-ID to the general population was based on the findings from the largest published NIMH epidemiological study of psychiatric disorders in children and adolescents $(\mathrm{N}=1,285$, ages 9-17 years) using DSM-III-R criteria (Shaffer et al. 1996).
Finally, the entire sample of FXS was compared to a sample of children $(\mathrm{N}=132$, ages 4-17 years) with WS assessed with the ADIS who had a similar range of cognitive ability (Leyfer et al. 2009).

More individuals with FXS+ID met criteria for an anxiety disorder than has been reported in other idiopathic ID groups (see Table 4). Rates of seven of the ten anxiety disorders included in the comparison were significantly higher in the FXS+ID group than the idiopathic ID group. In both the idiopathic ID and FXS + ID groups, specific and social phobias were the most commonly diagnosed anxiety disorders.

More than three-quarters of the FXS group with an average or borderline IQ (FSIQ > 70) met criteria for at least one anxiety disorder, compared to just $9.8 \%$ of the general population. The rates of all anxiety disorders in the FXS-ID group were significantly higher compared to the general population, with the exception of separation anxiety.

In comparison to a sample of individuals with WS with a similar level and range of cognitive abilities, the rate of specific phobia was similar. The rates of specific phobia, separation anxiety and PTSD were not significantly different between the FXS and WS groups. The number of individuals in the FXS group who met criteria for at least one anxiety disorder was significantly higher than the group with WS. Though the rate of GAD in WS was more than double the rate in the general population, it remained significantly lower compared to FXS. As expected, the rate of social phobia in FXS was much higher than in WS or ID.

\section{Discussion}

This study documented high rates of anxiety disorders in a large sample of individuals with FXS, using appropriate clinical diagnostic criteria for those with or without ID. Though the severity of anxiety and social withdrawal behaviors in FXS have been well described via parentreport behavior checklists, this is the first study to use a clinical tool for the assessment of DSM-IV diagnoses, among both males and females and across cognitive abilities. This study found similar results between a questionnaire previously developed and validated for use in ID and a semi-structured interview based on DSM-IV criteria, supporting both the appropriateness of the DSM-IV based interview used, as well as the specificity of both measures. The pervasiveness of anxiety in this population suggests a profound effect of the FMR1 gene mutation on anxiety symptoms.

The high percentage of females with FXS who met criteria for an anxiety disorder is in contrast to previous research. A study using a semi-structured diagnostic interview (SADS-L) to compare emotional problems in 
Table 4 Comparison of anxiety disorder prevalence rates among FXS with ID vs. Idiopathic ID, FXS without ID vs. General Population and FXS vs. Williams Syndrome

\begin{tabular}{|c|c|c|c|c|c|c|}
\hline \multirow[b]{2}{*}{ Anxiety type } & \multicolumn{2}{|c|}{ ID comparison $^{\mathrm{a}}$} & \multicolumn{2}{|c|}{ Non-ID comparison ${ }^{\mathrm{b}}$} & \multicolumn{2}{|c|}{ Genetic syndrome comparison $^{c}$} \\
\hline & $\mathrm{FXS}+\mathrm{ID}$ & $\mathrm{ID}^{\mathrm{d}}$ & FXS-ID & Gen. Pop. ${ }^{\mathrm{e}}$ & FXS & $W S^{f}$ \\
\hline Any anxiety disorder & $87.9 \%{ }^{*}$ & $10.5 \%$ & $76.3 \%^{*}$ & $9.8 \%$ & $82.5 \%{ }^{*}$ & $62.1 \%$ \\
\hline Separation anxiety & $10.3 \%$ & $1.9 \%$ & $13.5 \%{ }^{*}$ & $2.3 \%$ & $11.5 \%$ & $6.1 \%$ \\
\hline Social phobia & $32.8 \%{ }^{*}$ & $1.9 \%$ & $43.2 \%{ }^{*}$ & $4.5 \%$ & $36.5 \%{ }^{*}$ & $2.3 \%$ \\
\hline Specific phobia & $70.7 \%{ }^{*}$ & $6.8 \%$ & $42.9 \%{ }^{*}$ & $1.3 \%$ & $59.6 \%$ & $56.1 \%$ \\
\hline Panic Disorder & $6.9 \%$ & $\mathrm{~N} / \mathrm{A}$ & $2.9 \%$ & $\mathrm{~N} / \mathrm{A}$ & $5.4 \%$ & $\mathrm{~N} / \mathrm{A}$ \\
\hline -Agoraphobia & $1.7 \%$ & $0.2 \%$ & $0.0 \%$ & N/A & $1.1 \%$ & N/A \\
\hline +Agoraphobia & $5.2 \%{ }^{*}$ & $0.2 \%$ & $0.0 \%$ & $\mathrm{~N} / \mathrm{A}$ & $3.2 \%$ & $\mathrm{~N} / \mathrm{A}$ \\
\hline Agoraphobia & $17.9 \%{ }^{*}$ & $1.1 \%$ & $5.6 \%$ & $1.4 \%$ & $12.9 \%$ & N/A \\
\hline GAD & $21.4 \%{ }^{*}$ & $0.0 \%$ & $27.8 \%{ }^{*}$ & $3.1 \%$ & $23.7 \%{ }^{*}$ & $7.6 \%$ \\
\hline OCD & $26.8 \%{ }^{*}$ & $1.5 \%$ & $19.4 \%$ & N/A & $23.7 \%{ }^{*}$ & $1.5 \%$ \\
\hline PTSD & $1.7 \%$ & $0.0 \%$ & $8.6 \%$ & N/A & $4.3 \%$ & $1.5 \%$ \\
\hline Selective mutism & $27.6 \%$ & $\mathrm{~N} / \mathrm{A}$ & $22.2 \%$ & N/A & $25.3 \%$ & N/A \\
\hline
\end{tabular}

${ }^{\mathrm{a}}$ Control for multiple comparisons used, significant differences are $\mathrm{p}<.0025$

${ }^{\mathrm{b}}$ Control for multiple comparisons used, significant differences are $\mathrm{p}<.00417$

${ }^{\mathrm{c}}$ Control for multiple comparisons used, significant differences are $\mathrm{p}<.00357$

${ }^{\mathrm{d}}$ Dekker (2003) using DISC-IV-P, N=474, ${ }^{\mathrm{e}}$ Schaffer (1999) using DISC 2.3, N=1,285, ${ }^{\mathrm{f}}$ Leyfer (2009) N=132 using ADIS-IV

* Difference is significant at the corrected $(\mathrm{p})$ value

females with FXS, premutation carriers and controls found no difference between two 18-45 year old female control groups (familial or mothers of children with DD negative for FXS), premutation carriers or those with FXS in the lifetime incidence of anxiety disorders (Sobesky et al. 1994). This finding could be due in part to the genetic testing available at the time of the study, resulting in poor identification of the premutation range across groups and smaller sample size of females with FXS ( $\mathrm{N}=21)$, as well as the limited number of anxiety disorders (social phobia and GAD) assessed by the SADS-L. There have been disparate reports regarding the psychological problems experienced by females with FXS. However, this study affirms anxiety is experienced at similar rates as males, irrespective of an ASD or ID.

Selective mutism (SM) was the third most common disorder in the study, occurring in $25.3 \%$ of the sample. Although not formally classified as an anxiety disorder, SM is considered a severe form of social anxiety. DSM-IV criteria for SM can be summarized as a consistent failure to speak in specific social situations, despite speaking in other situations and is not better explained by a communication disorder or speech abnormalities. Further, social anxiety and avoidance (as part of social phobia) may be associated with SM and both diagnoses can be given. In the general population, prevalence estimates of SM are $0.47 \%-0.76 \%$ among children (Viana et al. 2009). Hagerman et al. (1999) documented the case of a 12-year old female with FXS who met criteria for SM. To our knowledge, there has been no other specific investigation of SM in FXS. Previous studies of psychiatric comorbidity in idiopathic autism have reported rates below the prevalence estimates of the general population and significantly lower than the rate found in the present study (de Bruin et al. 2007). Both autism status and language ability were taken into account before a diagnosis of SM was given. The vast majority of the sample met criteria for social anxiety and one-quarter met criteria for the additional diagnosis of SM. Though many parents of individuals with FXS and clinicians may observe selective talking in social situations, the high rate in this sample warrants replication.

Currently, the treatment of anxiety in FXS relies heavily on psychotropic medication, among which SSRIs are the most commonly prescribed (Berry-Kravis and Potanos 2004). Although over $80 \%$ of our sample met criteria for a DSM-IV anxiety disorder, just $27 \%$ were using SSRIs at the time of assessment. Cognitive-behavioral and pharmacological interventions are empirically validated for patients with anxiety disorders in the general population; however there has been little research on the utility of these methods in FXS.

There were no significant differences in the rates of anxiety relative to proband status or ASD, except for a higher rate of SM among those with an ASD. This suggests that the high rate of anxiety disorders in FXS reported here is unlikely to be associated with clinic referral bias, as the 
non-probands were generally not referred and seen only for research purposes. However, there was a trend towards more anxiety disorders and higher rates of specific phobia among those with an ASD. In a comparison of those with ID and a pervasive developmental disorder (ID+PDD) and those with ID without PDD (ID-PDD), Dekker and colleagues did not find an increased likelihood for any anxiety disorder, except OCD (Dekker and Koot 2003). Research examining comorbid diagnoses of anxiety and idiopathic autism have reported higher scores of anxiety and social worries in autism compared to controls (Gillott et al. 2001). A recent review concluded that anxiety is common in children and adolescents with autism spectrum disorders and may be a source of additional comorbidity (White et al. 2009). Other than SM, significant differences in anxiety related to autism were not found in this sample, confirming that anxiety is a pervasive problem in FXS.

The relationships found between the ADAMS, a measure normed and validated in ID and the ADIS, a DSM-IV based clinical interview, support the use of both measures in FXS. The relationship between the social anxiety disorders (social phobia and selective mutism) and the ADAMS Social Avoidance subscale demonstrate the specificity of each measure. The General Anxiety subscale of the ADAMS was significantly correlated with many of the ADIS subscales, suggesting it is a good measure of overall anxiety. The lack of significant relationship between the ADAMS Depression subscale and any of the ADIS anxiety diagnoses is notable considering the commonly found overlap in anxiety and depressive symptoms (Clark and Watson 1991; Brady and Kendall 1992; Stark and Laurent 2001).

In comparison to idiopathic ID, the general population, and WS, FXS had significantly higher rates of the majority of anxiety disorders. WS is another genetic disorder characterized by ID. The social behavior of FXS and WS differ: individuals with WS have a tendency for social engagement, while individuals with FXS are often characterized as socially withdrawn. The fact that FXS and WS have similar rates of ID and specific phobia but strikingly different rates of social phobia suggests that these two syndromes offer a unique opportunity to elucidate neuroanatomical and other biological mechanisms underlying social behavior.

Recently, there has been increased evidence of potential biological differences specific to FXS that may be related to anxiety. Research focusing on the biological bases of anxiety in FXS have found disruption of limbic-hypothalamic-pituitary-adrenal (L-HPA) axis activity and dysregulation of sympathetic-parasympathetic nervous system modulation in both human studies (Hessl et al. 2002, 2006) and animal-models of FXS (Markham et al. 2006; Spencer et al. 2005). Studies of sympathetic nervous system activity (Miller et al. 1999; Roberts et al. 2001; Farzin et al. 2009) have demonstrated enhanced reactivity to sensory stimuli that is inversely correlated with the level of FMRP (Miller et al. 1999). This hyperarousal may be associated with the high rates of anxiety observed in FXS. Further, the increased activity of metabotropic glutamate 5 receptors (mGluR5) and decreased activity of gammaaminobutyric acid (GABA) that have been documented in the animal models of FXS also appear to be related to anxiety and the use of targeted treatments in FXS may improve anxiety as has been shown in the mouse model of FXS (Berry-Kravis et al. 2009; Hagerman et al. 2009). Finally, recent neuroimaging studies of children and adults with FXS have implicated limbic structures such as the amygdala and insula (Hagan et al. 2008; Haas et al. 2009), which may mediate these hyper-responsive physiological systems and play an important role in emotion regulation, fear reactions and processing social stimuli. Future research linking these biological mechanisms associated with anxiety and hyperarousal to appropriate clinical measures of anxiety will have substantial implications for treatment and intervention design.

There were several important limitations of the study. First, although the ADIS has been used in prior studies of anxiety in ID, it has not been extensively validated for those with mental impairment. However, the high rates of anxiety disorders among individuals with FXS who do not have ID, the significant correlations with specific ADAMS subscales and the controlled comparison to WS ADIS data help to substantiate the application of this tool in the FXS population. Second, interviewers were not always blind to the FMR1 status of the participants. Third, we only assessed current disorders and are unable to report the lifetime incidence of anxiety in this sample. It is possible that some participants visited our facility seeking assistance with symptoms that were not salient in the past. Future studies that balance sample characteristics and recruitment approaches will validate the findings of this study. Finally, due to the nature of ID and its associated limitations on verbal communication and insight, we relied exclusively on parent report. Many anxiety symptoms are experienced internally and may not be reflected in external behavior observed by parents.

This study documents the pervasiveness of clinical anxiety in FXS. Among those with or without ID or an ASD, male or female, anxiety is a significant and frequently untreated problem in FXS. The striking prevalence of anxiety and previous findings of physiological dysregulation in FXS necessitates clinical assessment for anxiety disorders in FXS, along with other standard neuropsychological assessments. The results uncovered debilitating anxiety symptoms, suggesting a history of diagnostic overshadowing in FXS. With the advent and increased use of more appropriate measures of 
psychopathology in ID, the psychological problems in FXS and other ID populations can be more accurately measured and treated.

Acknowledgements The authors thank Susan Harris, Ashwini Mulgaonkar and Jennifer Yuhas for their contribution to this research. We are especially thankful for the participants and their families and their contribution to the understanding of fragile $\mathrm{X}$ syndrome. This work was supported by NIH grants MH77554, MH080025, and HD02274.

Financial disclosures Lisa Cordeiro and Elizabeth Ballinger report no conflicts of interest. Dr. David Hessl and Dr. Randi Hagerman report consultation with Novartis, Roche, and Seaside Therapeutics regarding fragile $\mathrm{X}$ treatment studies, as well as funding received from Seaside Therapeutics, Roche, and Neuropharm for clinical trials. Dr. Hagerman also reports funding from Johnson and Johnson and Forest pharmaceuticals for clinical trials.

Open Access This article is distributed under the terms of the Creative Commons Attribution Noncommercial License which permits any noncommercial use, distribution, and reproduction in any medium, provided the original author(s) and source are credited.

\section{References}

Bailey Jr DB, Raspa M, Olmsted M, Holiday DB. Co-occurring conditions associated with FMR1 gene variations: findings from a national parent survey. Am J Med Genet A. 2008;146A(16):2060-9.

Bassell GJ, Warren ST. Fragile X syndrome: loss of local mRNA regulation alters synaptic development and function. Neuron. 2008;60(2):201-14.

Berry-Kravis E, Potanos K. Psychopharmacology in fragile X syndrome-present and future. Ment Retard Dev Disabil Res Rev. 2004;10(1):42-8.

Berry-Kravis E, Hessl D, Coffey S, Hervey C, Schneider A, Yuhas $\mathrm{J}$, et al. A pilot open label, single dose trial of fenobam in adults with fragile X syndrome. J Med Genet. 2009;46 (4):266-71.

Bodden DH, Bogels SM, Nauta MH, De Haan E, Ringrose J, Appelboom C, et al. Child versus family cognitive-behavioral therapy in clinically anxious youth: an efficacy and partial effectiveness study. J Am Acad Child Adolesc Psychiatry. 2008;47(12):1384-94.

Bodden DH, Bogels SM, Muris P. The diagnostic utility of the Screen for Child Anxiety Related Emotional Disorders-71 (SCARED71). Behav Res Ther. 2009;47(5):418-25.

Brady EU, Kendall PC. Comorbidity of anxiety and depression in children and adolescents. Psychol Bull. 1992;111(2):244-55.

Clark LA, Watson D. Tripartite model of anxiety and depression: psychometric evidence and taxonomic implications. J Abnorm Psychol. 1991;100(3):316-36.

Cohen IL, Sudhalter V, Pfadt A, Jenkins EC, Brown WT, Vietze PM. Why are autism and the fragile-X syndrome associated? Conceptual and methodological issues. Am J Hum Genet. 1991;48(2):195-202.

Compton SN, Walkup JT, Albano AM, Piacentini JC, Birmaher B, Sherrill JT, et al. Child/Adolescent Anxiety Multimodal Study
(CAMS): rationale, design, and methods. Child Adolesc Psychiatr Ment Health. 2010;4:1.

Costello EJ, Mustillo S, Erkanli A, Keeler G, Angold A. Prevalence and development of psychiatric disorders in childhood and adolescence. Arch Gen Psychiatry. 2003;60(8):837-44.

Crawford DC, Meadows KL, Newman JL, Taft LF, Scott E, Leslie M, et al. Prevalence of the fragile $\mathrm{X}$ syndrome in AfricanAmericans. Am J Med Genet. 2002;110(3):226-33.

de Bruin EI, Ferdinand RF, Meester S, de Nijs PF, Verheij F. High rates of psychiatric co-morbidity in PDD-NOS. J Autism Dev Disord. 2007;37(5):877-86.

Dekker MC, Koot HM. DSM-IV disorders in children with borderline to moderate intellectual disability. I: prevalence and impact. J Am Acad Child Adolesc Psychiatry. 2003;42(8):915-22.

Dykens EM. Anxiety, fears, and phobias in persons with Williams syndrome. Dev Neuropsychol. 2003;23(1-2):291-316.

Eaton LF, Menolascino FJ. Psychiatric disorders in the mentally retarded: types, problems, and challenges. Am J Psychiatry. 1982;139(10):1297-303.

Einfeld SL, Tonge BJ, Rees VW. Longitudinal course of behavioral and emotional problems in Williams syndrome. Am J Ment Retard. 2001;106(1):73-81.

Esbensen AJ, Rojahn J, Aman MG, Ruedrich S. Reliability and validity of an assessment instrument for anxiety, depression, and mood among individuals with mental retardation. J Autism Dev Disord. 2003;33(6):617-29.

Farzin F, Rivera S, Hessl D. Brief report: visual processing of faces in individuals with fragile $\mathrm{X}$ syndrome: an eye tracking study. J Autism Dev Disord. 2009;39(6):946-52.

Fergusson DM, Horwood LJ, Lynskey MT. Prevalence and comorbidity of DSM-III-R diagnoses in a birth cohort of 15 year olds. J Am Acad Child Adolesc Psychiatry. 1993;32(6):1127-34.

Fernandez-Carvajal I, Walichiewicz P, Xiaosen X, Pan R, Hagerman PJ, Tassone F. Screening for expanded alleles of the FMR1 gene in blood spots from newborn males in a Spanish population. J Mol Diagn. 2009;11(4):324-9.

Freund LS, Reiss AL, Abrams MT. Psychiatric disorders associated with fragile $X$ in the young female. Pediatrics. 1993;91(2):321-9.

Gillott A, Furniss F, Walter A. Anxiety in high-functioning children with autism. Autism. 2001;5(3):277.

Gosch A, Pankau R. Personality characteristics and behaviour problems in individuals of different ages with Williams syndrome. Dev Med Child Neurol. 1997;39(8):527-33.

Gothelf D, Feinstein C, Thompson T, Gu E, Penniman L, Van Stone $\mathrm{E}$, et al. Risk factors for the emergence of psychotic disorders in adolescents with 22q11.2 deletion syndrome. Am J Psychiatry. 2007;164(4):663-9.

Haas B, Barnea-Goraly N, Lightbody A, Patnaik S, Hoeft F, Hazlett $\mathrm{H}$, et al. Early white-matter abnormalities of the ventral frontostriatal pathway in fragile X syndrome. Dev Med Child Neurol. 2009;51(8):593-9.

Hagan CC, Hoeft F, Mackey A, Mobbs D, Reiss AL. Aberrant neural function during emotion attribution in female subjects with fragile $\mathrm{X}$ syndrome. J Am Acad Child Adolesc Psych. 2008;47(12):1443.

Hagerman PJ. The fragile X prevalence paradox. J Med Genet. 2008;45(8):498-9.

Hagerman RJ, Hills J, Scharfenaker S, Lewis H. Fragile X syndrome and selective mutism. Am J Med Genet. 1999;83(4):313-7.

Hagerman RJ, Berry-Kravis E, Kaufmann WE, Ono MY, Tartaglia N, Lachiewicz A, et al. Advances in the treatment of fragile $\mathrm{X}$ syndrome. Pediatrics. 2009;123(1):378-90.

Harris SW, Hessl D, Goodlin-Jones B, Ferranti J, Bacalman S, Barbato I, et al. Autism profiles of males with fragile $\mathrm{X}$ syndrome. Am J Ment Retard. 2008;113(6):427-38.

Hessl D, Dyer-Friedman J, Glaser B, Wisbeck J, Barajas RG, Taylor A, et al. The influence of environmental and genetic factors on 
behavior problems and autistic symptoms in boys and girls with fragile X syndrome. Pediatrics. 2001;108(5):E88.

Hessl D, Glaser B, Dyer-Friedman J, Blasey C, Hastie T, Gunnar M, et al. Cortisol and behavior in fragile $\mathrm{X}$ syndrome. Psychoneuroendocrinology. 2002;27(7):855-72.

Hessl D, Glaser B, Dyer-Friedman J, Reiss AL. Social behavior and cortisol reactivity in children with fragile $\mathrm{X}$ syndrome. J Child Psychol Psychiatry. 2006;47(6):602-10.

Kessler RC, Berglund P, Demler O, Jin R, Merikangas KR, Walters EE. Lifetime prevalence and age-of-onset distributions of DSMIV disorders in the National Comorbidity Survey Replication. Arch Gen Psychiatry. 2005;62(6):593-602.

Lachiewicz AM. Abnormal behaviors of young girls with fragile $X$ syndrome. Am J Med Genet. 1992;43(1-2):72-7.

Lachiewicz A. Females with fragile X syndrome: a review of the effects of an abnormal gene. Ment Retard Dev Disabil Res Rev. 1995;1:292-7.

Lachiewicz AM, Dawson DV. Behavior problems of young girls with fragile X syndrome: factor scores on the Conners' Parent's Questionnaire. Am J Med Genet. 1994;51(4):364-9.

Lachiewicz AM, Dawson DV, Spiridigliozzi GA, McConkie-Rosell A. Arithmetic difficulties in females with the fragile $\mathrm{X}$ premutation. Am J Med Genet A. 2006;140(7):665-72.

Leyfer OT, Ruberg JL, Woodruff-Borden J. Examination of the utility of the Beck Anxiety Inventory and its factors as a screener for anxiety disorders. J Anxiety Disord. 2006a;20(4):444-58.

Leyfer OT, Woodruff-Borden J, Klein-Tasman BP, Fricke JS, Mervis CB. Prevalence of psychiatric disorders in 4 to 16 -year-olds with Williams syndrome. Am J Med Genet B Neuropsychiatr Genet. 2006b;141B(6):615-22.

Leyfer O, Woodruff-Borden J, Mervis CB. Anxiety disorders in children with Williams syndrome, their mothers, and their siblings: implications for the etiology of anxiety disorders. J Neurodevelopmental Disord. 2009;1(1):4-14.

Loesch DZ, Huggins RM, Hagerman RJ. Phenotypic variation and FMRP levels in fragile X. Ment Retard Dev Disabil Res Rev. 2004;10(1):31-41.

Lyneham HJ, Abbott MJ, Rapee RM. Interrater reliability of the Anxiety Disorders Interview Schedule for DSM-IV: child and parent version. J Am Acad Child Adolesc Psychiatry. 2007;46(6):731-6.

Markham J, Beckel-Mitchener A, Estrada C, Greenough W. Corticosterone response to acute stress in a mouse model of Fragile $\mathrm{X}$ syndrome. Psychoneuroendocrinology. 2006;31(6):781-5.

McGee R, Feehan M, Williams S, Partridge F, Silva PA, Kelly J. DSM-III disorders in a large sample of adolescents. J Am Acad Child Adolesc Psychiatry. 1990;29(4):611-9.

Miller LJ, McIntosh DN, McGrath J, Shyu V, Lampe M, Taylor AK, et al. Electrodermal responses to sensory stimuli in individuals with fragile X syndrome: a preliminary report. Am J Med Genet. 1999;83(4):268-79.

Murphy MM. A review of mathematical learning disabilities in children with fragile $\mathrm{X}$ syndrome. Dev Disabil Res Rev. 2009;15(1):21-7.

Myers BA, Pueschel SM. Psychiatric disorders in persons with Down syndrome. J Nerv Ment Dis. 1991;179(10):609-13.

Newcombe RG. Interval estimation for the difference between independent proportions: comparison of eleven methods. Stat Med. 1998a;17(8):873-90.

Newcombe RG. Two-sided confidence intervals for the single proportion: comparison of seven methods. Stat Med. 1998b;17(8):857-72.

Panichelli-Mindel SM, Flannery-Schroeder E, Kendall PC, Angelosante AG. Disclosure of distress among anxiety-disordered youth: differences in treatment outcome. J Anxiety Disord. 2005;19 (4):403-22.

Prasad SE, Howley S, Murphy KC. Candidate genes and the behavioral phenotype in 22q11.2 deletion syndrome. Dev Disabil Res Rev. 2008;14(1):26-34.

Reiss AL, Freund L. Behavioral phenotype of fragile X syndrome: DSM-III-R autistic behavior in male children. Am J Med Genet. 1992;43(1-2):35-46.

Reiss S, Valenti-Hein D. Development of a psychopathology rating scale for children with mental retardation. J Consult Clin Psychol. 1994;62(1):28-33.

Roberts JE, Boccia ML, Bailey DB, Hatton D, Skinner M. Cardiovascular indices of physiological arousal in boys with fragile X syndrome. Dev Psychobiol. 2001;39(2):107-23.

Ross CJ, Davis TM, Hogg DY. Screening and assessing adolescent asthmatics for anxiety disorders. Clin Nurs Res. 2007;16(1):524. discussion $5-8$

Shaffer D, Fisher P, Dulcan MK, Davies M, Piacentini J, SchwabStone ME, et al. The NIMH Diagnostic Interview Schedule for Children Version 2.3 (DISC-2.3): description, acceptability, prevalence rates, and performance in the MECA Study. Methods for the epidemiology of child and adolescent mental disorders study. J Am Acad Child Adolesc Psychiatry. 1996;35(7):865-77.

Silverman WK, Albano AM. Anxiety Disorders Interview Schedule (ADIS-IV) child/parent clinician manual. New York: Oxford University Press; 2004.

Silverman WK, Saavedra LM, Pina AA. Test-retest reliability of anxiety symptoms and diagnoses with the anxiety disorders interview schedule for DSM-IV: child and parent versions. J Am Acad Child Adolesc Psych. 2001;40(8):937-44.

Simon E, Bogels SM. Screening for anxiety disorders in children. Eur Child Adolesc Psychiatry. 2009;18(10):625-34.

Sobesky WE, Pennington BF, Porter D, Hull CE, Hagerman RJ. Emotional and neurocognitive deficits in fragile X. Am J Med Genet. 1994;51(4):378-85.

Spencer C, Alekseyenko O, Serysheva E, Yuva-Paylor L, Paylor R. Altered anxiety-related and social behaviors in the Fmr1 knockout mouse model of fragile $\mathrm{X}$ syndrome. Genes Brain Behav. 2005;4(7):420-30.

SPSS Statistics 17.0 Algorithms manual. Chicago, IL2007.

SPSS Statistics 17.0 Command Syntax Reference manual. Chicago, IL.2007.

Stark KD, Laurent J. Joint factor analysis of the children's depression inventory and the revised children's manifest anxiety scale. J Clin Child Psychol. 2001;30(4):552-67.

Sudhalter V, Cohen IL, Silverman W, Wolf-Schein EG. Conversational analyses of males with fragile X, Down syndrome, and autism: comparison of the emergence of deviant language. Am J Ment Retard. 1990;94(4):431-41.

Thaler NS, Kazemi E, Wood JJ. Measuring Anxiety in Youth with Learning Disabilities: Reliability and Validity of the Multidimensional Anxiety Scale for Children (MASC). Child Psychiatry Hum Dev. 2010;41(5):501-14.

Viana AG, Beidel DC, Rabian B. Selective mutism: a review and integration of the last 15 years. Clin Psychol Rev. 2009;29(1):57-67.

Waters AM, Mogg K, Bradley BP, Pine DS. Attentional bias for emotional faces in children with generalized anxiety disorder. J Am Acad Child Adolesc Psychiatry. 2008;47(4):435-42.

White SW, Oswald D, Ollendick T, Scahill L. Anxiety in children and adolescents with autism spectrum disorders. Clin Psychol Rev. 2009;29(3):216-29. 